\title{
Movement pattern of an ellipsoidal nanoparticle confined between solid surfaces: Theoretical model and molecular dynamics simulation
}

\author{
Junqin SHI ${ }^{1,2}$, Xiangzheng ZHU ${ }^{1}$, Kun SUN ${ }^{1}$, Liang FANG ${ }^{1,3, *}$ \\ ${ }^{1}$ State Key Laboratory for Mechanical Behavior of Materials, Xi'an Jiaotong University, Xi'an 710049, China \\ ${ }^{2}$ State Key Laboratory of Solidification Processing, Center of Advanced Lubrication and Seal Materials, Northwestern \\ Polytechnical University, Xi'an 710072, China \\ ${ }^{3}$ School of Mechanical and Electrical Engineering, Xiamen University Tan Kah Kee College, Zhangzhou 363105, China \\ Received: 13 March 2020 / Revised: 26 April 2020 / Accepted: 07 May 2020 \\ (C) The author(s) 2020 .
}

\begin{abstract}
The movement pattern of ellipsoidal nanoparticles confined between copper surfaces was examined using a theoretical model and molecular dynamics simulation. Initially, we developed a theoretical model of movement patterns for hard ellipsoidal nanoparticles. Subsequently, the simulation indicated that there are critical values for increasing the axial ratio, driving velocity of the contact surface, and lowering normal loads (i.e., $0.83,15 \mathrm{~m} / \mathrm{s}$, and $100 \mathrm{nN}$ under the respective conditions), which in turn change the movement pattern of nanoparticles from sliding to rolling. Based on the comparison between the ratio of arm of force $(e / h)$ and coefficient of friction $(\mu)$, the theoretical model was in good agreement with the simulations and accurately predicted the movement pattern of ellipsoidal nanoparticles. The sliding of the ellipsoidal nanoparticles led to severe surface damage. However, rolling separated the contact surfaces and thereby reduced friction and wear.
\end{abstract}

Keywords: movement pattern; friction and wear reduction; ellipsoidal nanoparticle additive; molecular dynamics simulation

\section{Introduction}

Lubrication is an important method to reduce friction and wear, and it improves the durability and reliability of materials and reduces energy consumption [1, 2]. Among the different lubrication regimes, the boundary lubrication exhibits high friction and wear behavior [3]. Currently, the addition of friction modifier additives (i.e., organic friction modifiers and nanoparticles) to tribology and lubrication systems is considered as a useful method for friction and wear reduction [2-5]. Given their thermal stability at high temperatures, nanoparticles can be popular solid lubricating additives and can play important roles in emission reduction and improving fuel economy [5].

Different types of nanoparticles, such as metal, metal oxide, metal carbonate, fullerenes, silica, and carbon compound, have been developed as solid lubricants and lubricant modifiers for friction modification and wear resistance [3, 6-14]. The tribological properties of metal nanoparticle additives (like $\mathrm{Fe}, \mathrm{Cu}, \mathrm{Ni}$, and $\mathrm{Co}$ ) in base lubricants indicate that they play a significant role in decreasing friction and wear by forming a tribo-layer [6-9]. Metal oxide nanoparticles, such as $\mathrm{CuO}, \mathrm{ZrO}_{2}$, and $\mathrm{ZnO}$, exhibit good friction and wear-reducing performance owing to the tribo-sintering process

* Corresponding author: Liang FANG, E-mail: fang1@xjtu.edu.cn 


$$
\begin{aligned}
& \text { Nomenclature } \\
& e \\
& h \\
& F, F_{1}, F_{2} \\
& N, N_{1}, N_{2} \\
& \mu \\
& a, b, c \\
& \gamma \\
& H \\
& \alpha \\
& \theta \\
& \alpha \theta \\
& \beta \\
& R
\end{aligned}
$$

\author{
Resistant arm of force \\ Driving arm of force \\ Friction force \\ Normal force \\ Coefficient of friction \\ Semiaxis of ellipsoidal nanoparticle \\ Rotation angle of ellipsoid \\ Material hardness \\ Elastic recovery coefficient \\ Indented angle \\ Elastic recovery angle \\ Angle of ellipse parametric expression \\ Radius of curvature
}

[10-12]. For example, it was considered that the deposition of $\mathrm{CuO}$ nanoparticles on a worn surface decreases the shearing stress and thus improves the tribological properties [13]. Carbon-based nanoparticles are being considered as potential boundary lubricant additives because of their high structural and chemical stability and environmentally friendly features [5, 14, 15]. Gupta et al. [16, 17] demonstrated a reduction in material wear by using fullerene (C60) nanoparticles. The reduction in material wear was owing to the formation of the transfer-film in the contact area or the "tiny ball bearings" effect of C60 clusters. Furthermore, the use of nanodiamonds as additives in sunflower oil and base oil decreased the worn scar depths by $43.3 \%$ and $62.1 \%$, respectively, when compared with pure oil lubrication [13]. Xu et al. [18] indicated that the friction and wear reduction was attributed to the ball-bearing effect and surface polishing action of diamond nanoparticles confined between the rubbing faces. Based on previous studies, the friction and wear reduction of nanoparticles are usually attributed to the rolling effect, tribofilm formation, mending effect, and polishing effect $[3,5,13,14]$. However, they significantly depend on the movement pattern of nanoparticles [4, 14, 19-21].

Furthermore, it is important to note that the lubrication and tribology behaviors of nanoparticles depend on many factors such as the individual features of the nanoparticles (size, shape, and physicochemical nature), material properties, and external tribological conditions [2, 4, 13, 22]. In boundary lubrication and mixed lubrication regimes, the shape of nanoparticles, moving status of rubbing faces, and external pressure significantly affect the movement pattern of nanoparticles confined between solid surfaces. This in turn is related to the friction mechanisms [23-26]. However, to date, there are no accurate theoretical models to predict the movement pattern of nanoparticle additives with non-regular shapes. Thus, it is not possible to accurately evaluate their lubrication and tribology behaviors, although Zhang et al. [24, 27] developed a theoretical prediction model for spherical nanoparticles. On the other hand, there is significant uncertainty concerning the relationship between the friction and wear reduction mechanisms and tribological conditions.

Therefore, to predict the movement pattern of non-spherical nanoparticles and illustrate their friction and wear reduction behaviors and the corresponding mechanisms, we created a mathematical model of the ratio of arm of force $(e / h, e$ is the resistant arm of force and $h$ is the driving arm of force) and compared it with that of coefficient of friction $(\mu)$. Subsequently, we implemented a nonequilibrium molecular dynamics (NEMD) simulation. A diamond ellipsoid was selected to model a hard and nonspherical nanoparticle additive. The shape of the nanoparticle, normal load, and relative velocity of solid surfaces were modified to validate the theoretical model and reveal the movement of the nanoparticles. This study will aid in providing 
insights for the nanoscale lubrication and tribology behavior of nanoparticles as lubricating additives confined between asperities. It is important to note that it is difficult to obtain these insights from experiments.

\section{Mathematical model of movement criterion for ellispoidal nanoparticles}

\subsection{Creation of movement criterion for ellispoidal nanoparticle}

In real lubrication or tribology systems, the movement pattern of nanoparticles varies and includes sliding, rolling, and/or their combination under the effects of tribological conditions such as material hardness, particle shape, and external load. It is important to predict the movement pattern of nanoparticles, and thereby explore the friction and wear behaviors. Fang et al. [28] proposed a criterion to predict the movement pattern of particles in macroscale and nanoscale, which is simply expressed in Eqs. (S1)(S4) in Electronic Supplementary Material (ESM). As shown in Fig. 1(a), the particle comes in contact with two surfaces in a small area wherein friction and normal forces act. The concentrated horizontal friction forces and normal forces are expressed with $F_{1}, F_{2}$, and $N_{1}, N_{2}$, such that the equal force correlations are $F=F_{1}=F_{2}$ and $N=N_{1}=N_{2}$ under equilibrium conditions. Conversely, when the particle rolls the torque $F \times h$ driving particle to roll, it should be higher than the torque $N \times e$ resisting particle rolling (that is $F \times h>N \times e$ ), and vice versa. Thus, the particle should roll if the $e / h$ is lower than $\mu$ defined by $F / N$ (i.e., $e / h<\mu$ for rolling). However, it should slide if $e / h$ is larger than $\mu$ (i.e., $e / h>\mu$ for sliding). It is important to note that this criterion is applicable to nearly all shapes of particles. However, it was created for spherical particles. Hence, the movement pattern of the particles can be predicted by comparing $e / h$ and $\mu$. The $e / h$ for spherical particles is shown in Eqs. (S5)-(S7) in ESM [28]. However, the shape of the nanoparticle is typically not regular and can influence the particles' movement pattern in boundary lubrication. Fang et al. [23, 28] assumed an ellipsoidal particle shape in the first approximation to model real particle contours in threebody abrasion. In this study, we adopted this ellipsoidal nanoparticle to model real nanoparticle additives in the lubrication or tribology system and formulated the $e / h$ expression to predict the movement pattern. As shown in Fig. 1, we used a sphere to replace the ellipsoid (with semi-axes $a, b$, and $c, a=c$ ) at the contact area. The sphere and ellipsoid exhibit the same curvature at contact point A. To simplify the process, three hypotheses were proposed as follows: 1) Nanoparticle is rigid

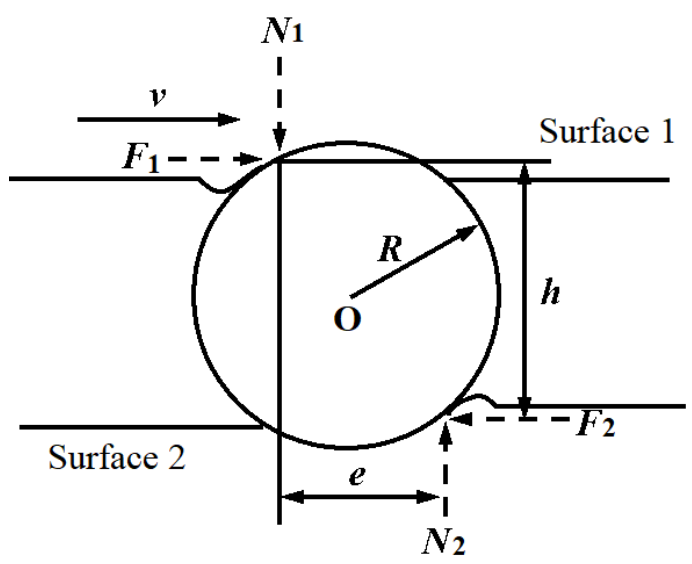

(a)

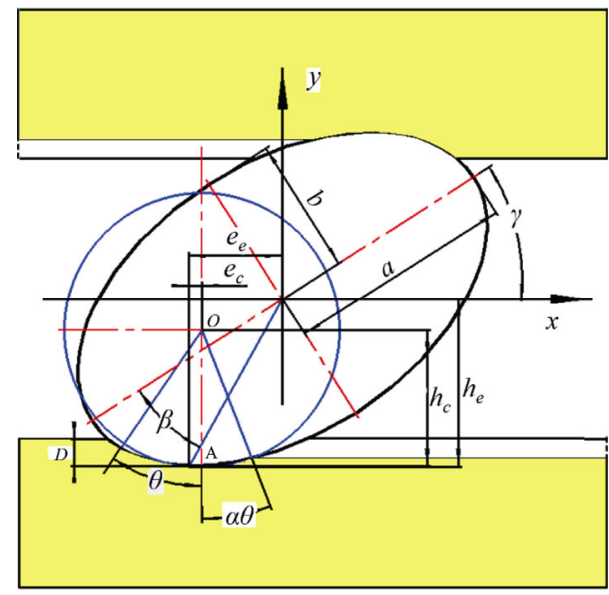

(b)

Fig. 1 Schematic illustrations of (a) force analysis for a spherical particle, and (b) the geometric parameters for ellipsoidal nanoparticle. The coordinate of point $\mathrm{A}$ is denoted by $\left(x_{1}, y_{1}\right)$, and the coordinate of point $\mathrm{O}$ is denoted by $\left(x_{01}, y_{01}\right)$. Symbol codes: $\theta$, indented angle; $\alpha$, elastic recovery coefficient; $\alpha \theta$, elastic recovery angle; $\beta$, angle of ellipse parametric expression; $\gamma$, rotation angle; $e_{\mathrm{e}}$ and $h_{\mathrm{e}}$, arm of force for ellipsoid; $e_{\mathrm{c}}$ and $h_{\mathrm{c}}$, arm of force for circle. 
and its mass is not considered; 2) nanoparticle cannot rotate in $\mathrm{XOZ}$ plane, but can rotate in $\mathrm{XOY}$ plane; 3 ) friction and normal forces are distributed uniformly on the contact surface.

The parametric expression of an ellipse is shown in Eq. (1), where $(x, y)$ denotes the coordinate of a point on the ellipse. Based on the first and second derivatives of the parametric expression, we can obtain the radius of curvature $(R)$ and the center coordinates of curvature circle, $\left(x_{0}, y_{0}\right)$, as described by Eqs. (2) and (3), respectively.

$$
\begin{gathered}
\left\{\begin{array}{l}
x=a \cos \beta \\
y=b \sin \beta
\end{array}\right. \\
R=\frac{\left[\left(a^{2}-b^{2}\right) \sin \beta^{2}+b^{2}\right]^{\frac{3}{2}}}{a b} \\
\left\{\begin{array}{l}
x_{0}=\frac{\cos \beta^{3}\left(a^{2}-b^{2}\right)}{a} \\
y_{0}=\frac{\sin \beta^{3}\left(b^{2}-a^{2}\right)}{b}
\end{array}\right.
\end{gathered}
$$

When the rotation angle of an ellipsoid is $\gamma$, as shown in Fig. 1, the new ellipse expression can be described by Eq. (4), where $\left(x_{1}, y_{1}\right)$ replaces $(x, y)$ as the coordinate of a point in the new ellipse, i.e., point A. Hence, the new center coordinates $\left(x_{01}\right.$, $y_{01}$ ) of curvature circle can be described by Eq. (5) as follows:

$$
\begin{gathered}
\left\{\begin{array}{l}
x_{1}=a \cos \beta \cos \gamma-b \sin \beta \sin \gamma \\
y_{1}=a \cos \beta \sin \gamma+b \sin \beta \cos \gamma
\end{array}\right. \\
\left\{\begin{array}{l}
x_{01}=\frac{\cos \beta^{3}\left(a^{2}-b^{2}\right)}{a} \cos \gamma-\frac{\sin \beta^{3}\left(b^{2}-a^{2}\right)}{b} \sin \gamma \\
y_{01}=\frac{\cos \beta^{3}\left(a^{2}-b^{2}\right)}{a} \sin \gamma+\frac{\sin \beta^{3}\left(b^{2}-a^{2}\right)}{b} \cos \gamma
\end{array}\right.
\end{gathered}
$$

Based on the rotating trajectory of curvature circle, the $e$ and $h$ can be obtained as shown in Eqs. (6) and (7), respectively. Hence, the $e / h$ expression is obtained in Eq. (8) as follows:

$$
\begin{gathered}
e_{\mathrm{e}}=e_{\mathrm{c}}+x_{01} \operatorname{sign}\left(x_{1}\right) \\
h_{\mathrm{e}}=h_{\mathrm{c}}+y_{01} \operatorname{sign}\left(y_{1}\right) \\
e_{\mathrm{e}} / h_{\mathrm{e}}=\frac{e_{\mathrm{c}}+x_{01} \operatorname{sign}\left(x_{1}\right)}{h_{\mathrm{c}}+y_{01} \operatorname{sign}\left(y_{1}\right)}
\end{gathered}
$$

By substituting Eqs. (S5) and (S6) (ESM), (4), and (5) into Eq. (8), the $e / h$ expression for an ellipsoidal nanoparticle moving along the $x$-direction is as follows:

$$
e_{\mathrm{e}} / h_{\mathrm{e}}=\frac{\frac{8\left[\left(a^{2}-b^{2}\right) \sin \beta^{2}+b^{2}\right]^{\frac{3}{2}}\left[\sin \theta^{3}-\sin (\alpha \theta)^{3}\right]}{3 \pi a b\left(\sin \theta^{2}+\sin (\alpha \theta)^{2}\right)}+\left[\frac{\cos \beta^{3}\left(a^{2}-b^{2}\right)}{a} \cos \gamma-\frac{\sin \beta^{3}\left(b^{2}-a^{2}\right)}{b} \sin \gamma\right] \operatorname{sign}\left(x_{1}\right)}{\frac{4\left[\left(a^{2}-b^{2}\right) \sin \beta^{2}+b^{2}\right]^{\frac{3}{2}} \sin (\theta)^{3}}{3 a b[\theta-\sin \theta \cos \theta]}+\left[\frac{\cos \beta^{3}\left(a^{2}-b^{2}\right)}{a} \sin \gamma+\frac{\sin \beta^{3}\left(b^{2}-a^{2}\right)}{b} \cos \gamma\right] \operatorname{sign}\left(y_{1}\right)}
$$

and

$$
\theta=\arccos \left\{\sqrt{1-\frac{N a^{2} b^{2}}{\pi H\left[\left(a^{2}-b^{2}\right) \sin \beta^{2}+b^{2}\right]}}\right\}
$$

where $\gamma$ denotes the rotation angle of the ellipsoid, $N$ denotes the normal load, and $H$ denotes material hardness.

\subsection{Discussion of $e / h$ expression}

Given that $a=b$ in Eq. (9), the ellipsoidal nanoparticle becomes a spherical nanoparticle. Thus, the $e / h$ expression for spherical nanoparticles can be obtained as described in Eq. (11). This expression is similar to that proposed by Sun et al. [27], and thereby indicating that Eq. (9) is correct at the specific condition.

$$
\begin{aligned}
& e_{\mathrm{c}} / h_{\mathrm{c}}= \frac{8 b\left[\sin \theta^{3}-\sin (\alpha \theta)^{3}\right]}{3 \pi\left[\sin \theta^{2}+\sin (\alpha \theta)^{2}\right]} \\
& \frac{4 b \sin \theta^{3}}{3[\theta-\sin \theta \cos \theta]} \\
&=\frac{2\left[\sin \theta^{3}-\sin (\alpha \theta)^{3}\right](\theta-\sin \theta \cos \theta)}{\pi\left[\sin \theta^{2}+\sin (\alpha \theta)^{2}\right] \sin \theta^{3}}
\end{aligned}
$$




$$
=\frac{2(\theta-\sin \theta \cos \theta)}{\pi\left(\sin \theta^{2}+\sin (\alpha \theta)^{2}\right)}\left\{1-\left[\frac{\sin (\alpha \theta)}{\sin \theta}\right]^{3}\right\}
$$

Concerning the $e / h$ expression of ellipsoidal nanoparticle in the lubrication system, $e / h$ is related to $\gamma, \theta, \alpha \theta$, and $\beta$ (as displayed in the list of symbols) in parametric expression. In Fig. 2, the correlations indicate that these angles exhibit important effects on the $e / h$ value. This further affects the relationship between $e / h$ and $\mu$. The $\theta$, $\alpha \theta$, and $\beta$ are related to the normal load, material properties, and shape of nanoparticle, respectively. Hence, they affect the movement pattern of nanoparticles in the lubrication and tribology process.

\section{NEMD simulation}

To validate the mathematical model for ellipsoidal nanoparticles and reveal the wear behavior, NEMD simulations with classical molecular dynamics code large-scale atomic/molecular massively parallel simulator (LAMMPS) [30] were performed. In all the simulations, two monocrystalline $\mathrm{Cu}$ slabs, with dimensions corresponding to $32.6 \mathrm{~nm} \times$ $20.2 \mathrm{~nm} \times 14.9 \mathrm{~nm}$ along $x$-[100], $y$-[010], and $z$-[001] crystal orientations, were used to represent the asperity contact, as shown in Fig. 3. Each $\mathrm{Cu}$ slab included boundary, thermostat, and Newtonian zones. The boundary zone was kept rigid to provide structural stability, the thermostat zone was set at a constant temperature of $300 \mathrm{~K}$ to mimic heat dissipation, and atoms in the Newtonian zone were allowed to freely move based on Newton's laws of motion. The ellipsoidal diamond nanoparticle (with semi-axes $a, b$, and $c$ along $x-, y$-, and $z$-directions, respectively) was confined between the two slabs, and its axial ratio $(b / a$, where $a=c=3 \mathrm{~nm})$ was varied to reflect the change in shape of nanoparticles, as listed in Table 1. Furthermore, the axial ratio was adjusted by only changing the value of $b$, and thereby signifying that the change in the nanoparticle shape is accompanied by variation in the volume of the nanoparticle $[23,25]$. To simplify the model, the lubricating fluids were omitted because most of them were squeezed out from the contact area of asperities [14, 31, 32].

The embedded atom method (EAM) potential [33] was adopted to describe the interactions between $\mathrm{Cu}$ atoms and the function is described in Eq. (12), where $F^{\prime}$ denotes the embedding energy, $\rho$ denotes the atomic electron density, $\varphi$ denotes a pair of potential interactions, and $k$ and $l$ denote the element types of atoms $i$ and $j$, respectively. The interactions between $\mathrm{Cu}$ atoms and $\mathrm{C}$ atoms of the diamond nanoparticles were modeled using the Morse potential [34], and the corresponding parameters were validated [25, 26]. The Morse function is shown in Eq. (13), where $D_{0}$ denotes the cohesion energy, $\delta$ denotes the elastic modulus, $r$ and $r_{0}$ denote the instantaneous and equilibrium distance between the two atoms, respectively. The interactions between $\mathrm{C}$ atoms of a diamond nanoparticle were omitted due to its rigid body [25]. All the parameters are shown in Table 1 [25, 35-39].

$$
E_{i}=F_{k}^{\prime}\left(\sum_{j \neq i} \rho_{l}\left(r_{i j}\right)\right)+\frac{1}{2} \sum_{j \neq i} \phi_{k l}\left(r_{i j}\right)
$$
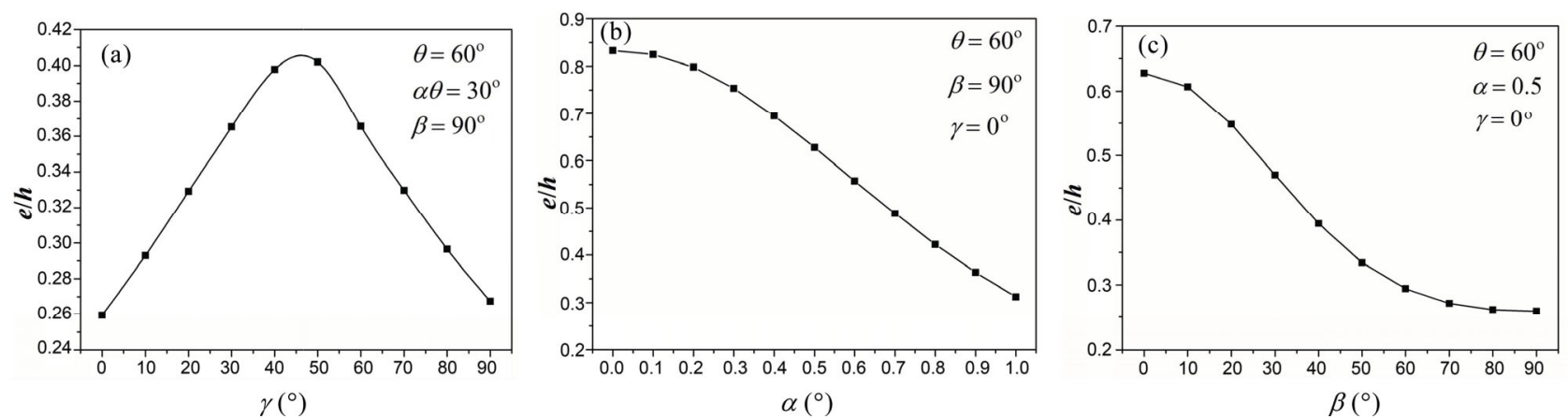

Fig. $2 e / h$ vs. $\gamma, \alpha$, and $\beta$. 


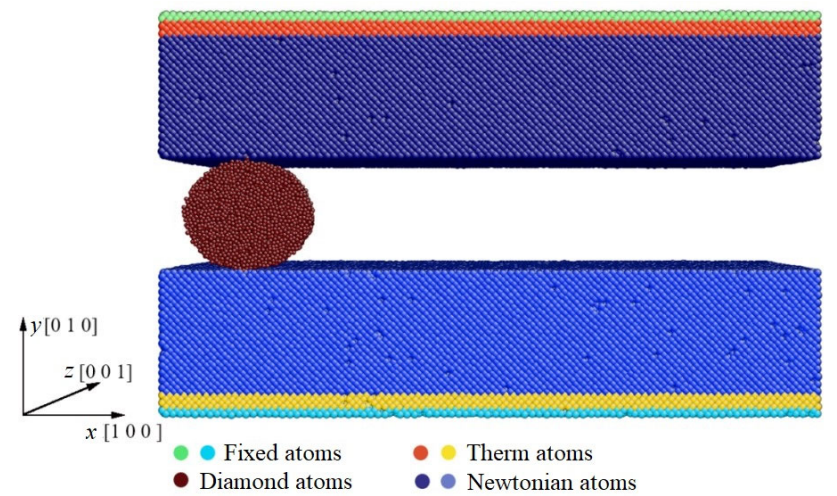

Fig. 3 Schematic of the molecular dynamics simulation model in a vertical cross-section.

Table 1 Parameters used in the MD simulations.

\begin{tabular}{cc}
\hline Property & Parameters \\
\hline Each Cu slab & Monocrystalline $\mathrm{Cu}, 575,640 \mathrm{atoms}$, \\
$32.6 \mathrm{~nm} \times 20.2 \mathrm{~nm} \times 14.9 \mathrm{~nm}$ \\
Ellipsoidal & $\begin{array}{c}\text { Diamond, rigid, } a=c=3 \mathrm{~nm} \text {, axial } \\
\text { nanoparticle }\end{array}$ \\
$\begin{array}{c}\text { Potential for } \\
\mathrm{Cu}-\mathrm{Cu}\end{array}$ & $\mathrm{EAM}, 0.70,0.83$, and 0.90 \\
Potential for & Morse: $D_{0}=0.01 \mathrm{eV}, \alpha=1.77 \mathrm{~nm}^{-1}$, and \\
$\mathrm{Cu}-\mathrm{C}$ & $r_{0}=0.22 \mathrm{~nm}$ \\
Normal load & $60,80,100$, and $110 \mathrm{nN}$ \\
Velocity of upper & $10,20,30$, and $50 \mathrm{~m} / \mathrm{s}$ \\
slab & $1 \mathrm{fs}$ \\
Timestep & $300 \mathrm{~K}$ \\
Temperature &
\end{tabular}

$$
E=D_{0}\left[e^{-2 \delta\left(r-r_{0}\right)}-2 e^{-\delta\left(r-r_{0}\right)}\right]
$$

In the NEMD simulation process, the system was initially optimized to obtain the local minimum energy and configuration at the canonical ensemble (NVT, constant atom number $N$, volume $V$, and temperature $T$ in simulated system) for 100 ps. Subsequently, the normal load was exerted on the boundary zone of the upper slab along the $y$-direction, while the boundary zone of the lower slab was fixed. Finally, the upper slab was derived to move along the $x$-direction at a constant velocity. All simulations were performed at the microcanonical ensemble (NVE, energy $E$ in simulated system), and the motion of atoms was integrated using the Velocity-Verlet algorithm with a time step of $1 \mathrm{fs}$. The Langevin thermostat was in the thermostat zone, and periodic boundary conditions were used along $x$ - and $z$-directions. The $\mu$ was obtained by calculating the average of the two coefficients of friction between the nanoparticle and the two solid surfaces.

\section{Results and discussion}

The NEMD simulations were performed to explore the change in the movement pattern of hard nanoparticle as a lubricant additive. Furthermore, the resultant friction and wear behaviors under different tribological conditions were also characterized. Figure 4 shows the instantaneous configurations of each ellipsoidal nanoparticle confined between monocrystalline $\mathrm{Cu}$ slabs at a normal load of $80 \mathrm{nN}$ and driving velocity of $50 \mathrm{~m} / \mathrm{s}$. It is observed that the nanoparticles, with an axial ratio of 0.50 and 0.70 , nearly maintained their initially lying state between two contact surfaces during the entire moving process. This denotes that the nanoparticles maintained a sliding movement pattern under the driving of the upper slab. The status of the nanoparticles with an axial ratio of 0.83 and 0.90 , which exhibits a shape closer to a sphere, varied at different moments. This implies that the nanoparticles maintained a rolling movement pattern. By combining Eq. (9) and the simulated results in Fig. 5, the calculated $e / h$ is always higher than $\mu$ because the axial ratio is less than 0.83 . This implies that $F \times h$ is lower than $N \times e$, and vice versa. This indicates that the ellipsoidal nanoparticles can slide if the axial ratio is less than 0.83 and roll if the axial ratio is higher or equal to 0.83 based on the criterion of the movement pattern.

Figure 6 shows the instantaneous configurations of the ellipsoidal nanoparticle with an axial ratio of 0.83 under different normal loads and a constant driving velocity of $50 \mathrm{~m} / \mathrm{s}$. Figure 6 indicates that the ellipsoidal nanoparticles rolled under the driving of the upper slab at normal loads of 60,80 , and $100 \mathrm{nN}$. However, sliding movement occurred as the normal load increased to $110 \mathrm{nN}$. It is important to note that the sliding nanoparticle experienced initial rolling from 0 to $60 \mathrm{ps}$, and thus it did not exhibit sliding alone. The calculated average $e / h$ value and $\mu$ validate the movement pattern of the ellipsoidal nanoparticle, as shown in Fig. 7. Similarly, Fig. 8 shows the ellipsoidal nanoparticle with an 


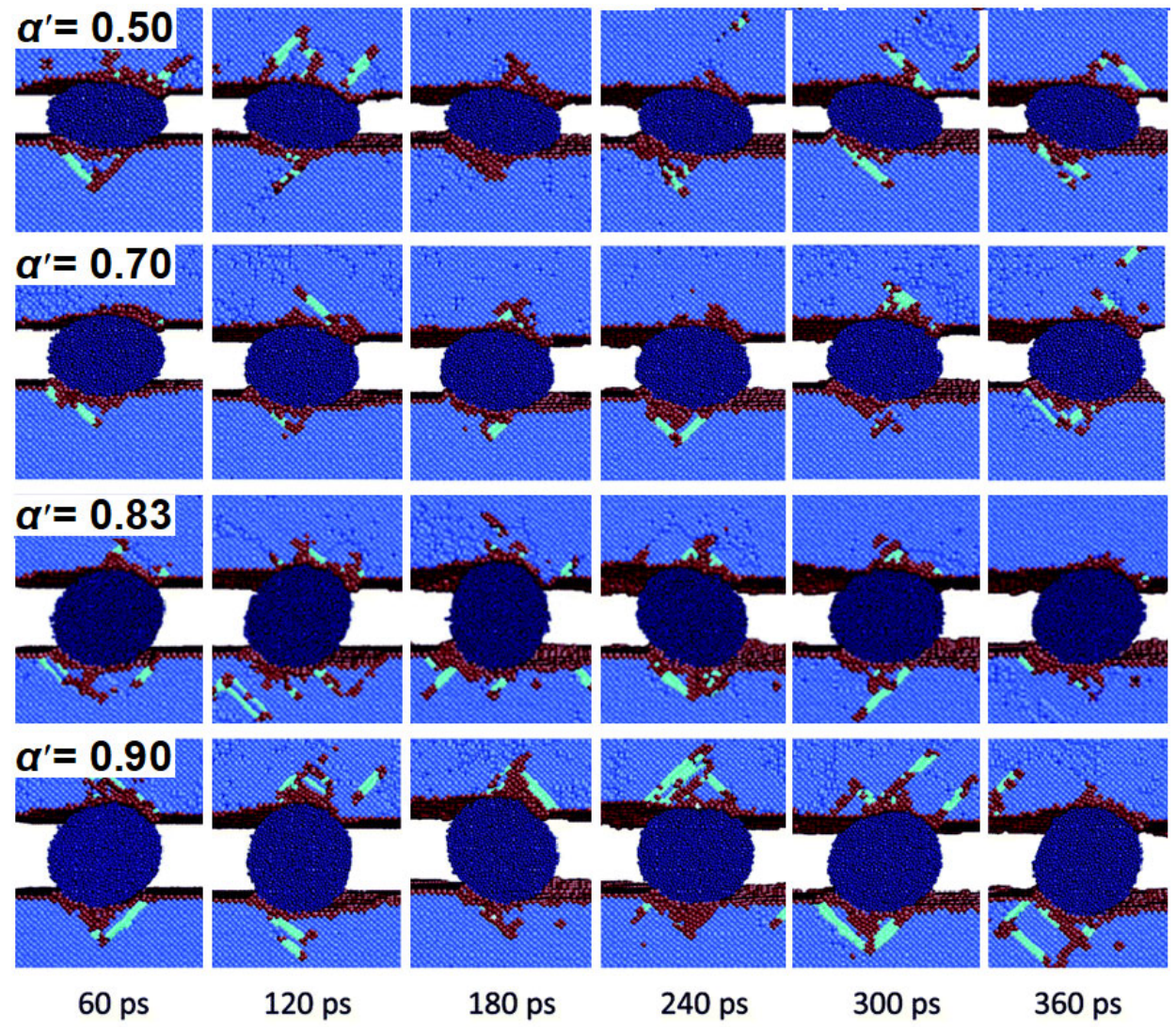

Fig. 4 Movement configurations of nanoparticle with variation in axial ratio $\alpha^{\prime}$. Color code: dark blue, nanoparticles; sky blue, defect-free $\mathrm{Cu}$; cyan, stacking fault; dark brown, $\mathrm{Cu}$ surface atoms.

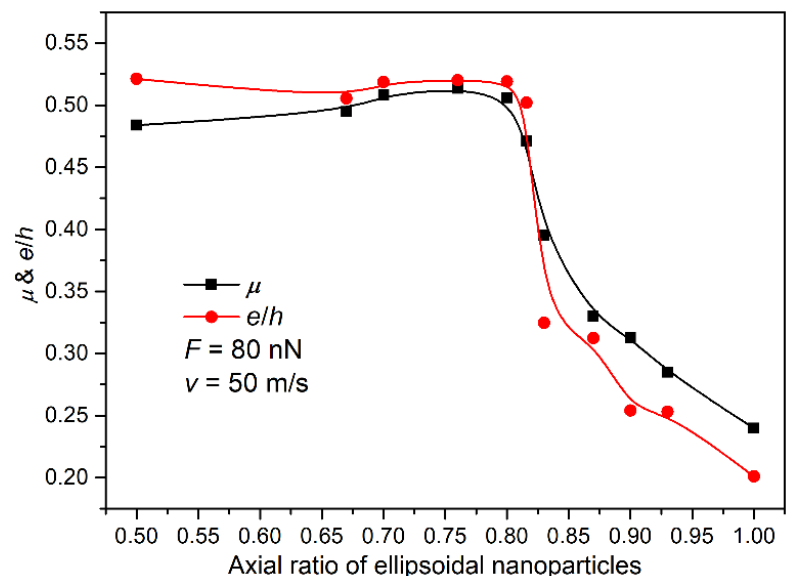

Fig. 5 Comparison of $\mu$ and $e / h$ concerning the axial ratio.

axial ratio of 0.83 , which maintained a nearly lying status with a small rotation angle under the driving velocity of $10 \mathrm{~m} / \mathrm{s}$. However, the nanoparticle exhibited a relatively high angle rotation during the first 5 $\mathrm{nm}$ and then maintained its status of sliding as the driving velocity increased to $15 \mathrm{~m} / \mathrm{s}$. Furthermore, as the driving velocity increased to $30 \mathrm{~m} / \mathrm{s}$, the nanoparticle exhibited a rolling movement pattern. The comparison of the calculated average $e / h$ value and $\mu$ (Fig. 9) confirms the various movement patterns of the ellipsoidal nanoparticles, as depicted in the configurations. The critical driving velocity is approximately 17 $\mathrm{m} / \mathrm{s}$ under the given tribology conditions.

The movement pattern of the ellipsoidal nanoparticles is significantly affected by the lubrication and tribology conditions. For example, the movement pattern turned from sliding to rolling as the axial ratio and driving velocity were increased and the normal load was decreased. As mentioned in Section 2.2, the $e / h$ value is related to $\gamma, \theta, \alpha \theta$, and $\beta$ in parametric expression. Furthermore, the $\theta$ exhibited a certain relationship with normal load, material hardness, and shape of the nanoparticle, as shown by Eq. (10), and $\beta$ is related to the shape of nanoparticles, the $\alpha$ is related to the elastic property of the material, and the $\gamma$ is related to the normal load and shape of the nanoparticles. Therefore, if the relationships between the $\gamma$ and normal load and 


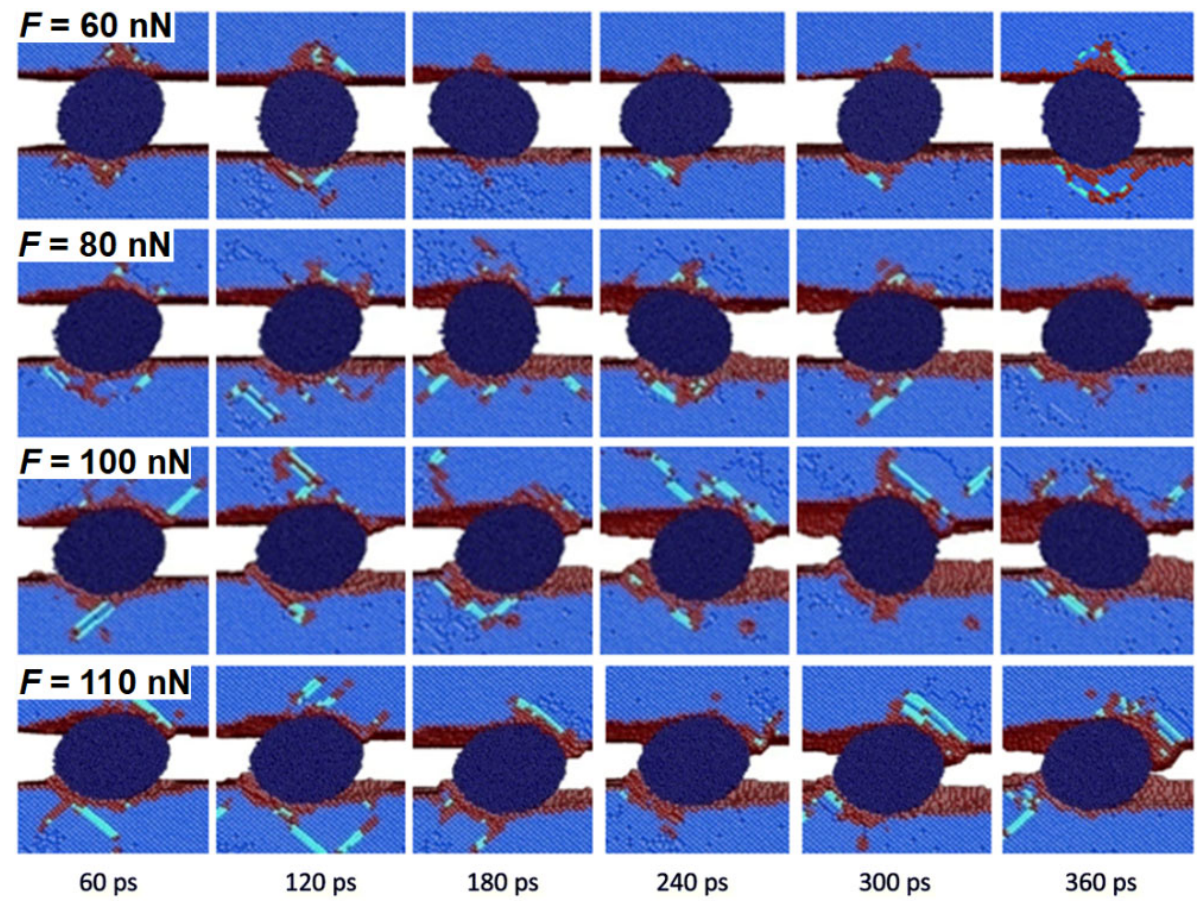

Fig. 6 Movement configurations of nanoparticle with an axial ratio of 0.83 under different normal loads.

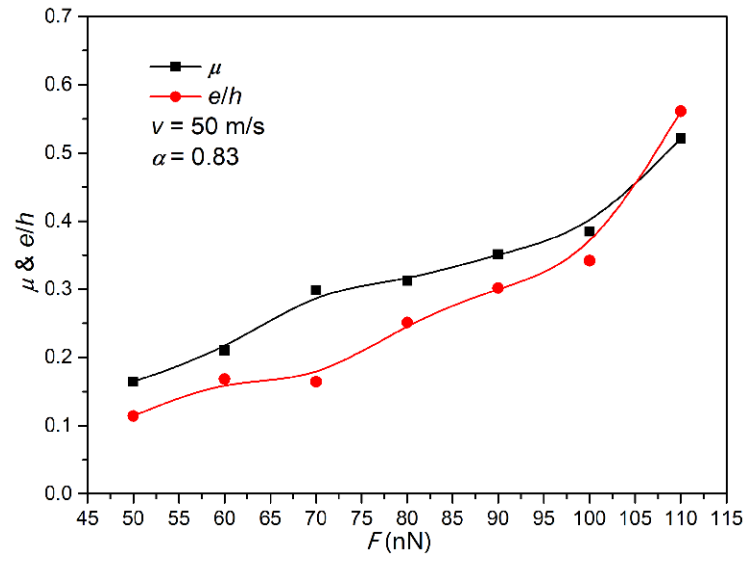

Fig. 7 Comparison between $\mu$ and $e / h$ under different normal loads.

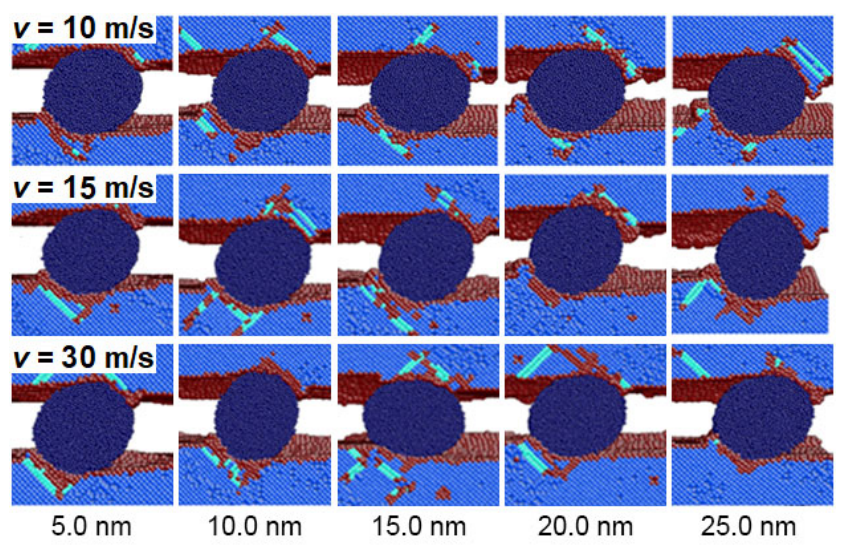

Fig. 8 Movement configurations of nanoparticle under different driving velocities.

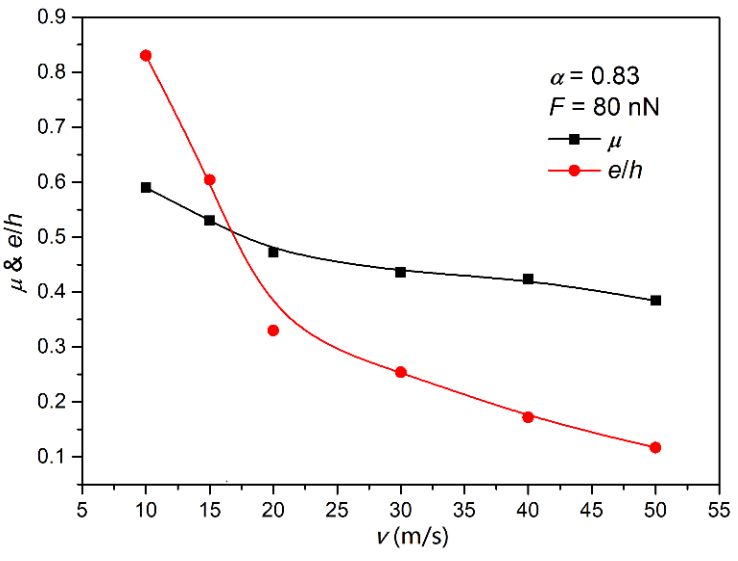

Fig. 9 Comparison between $\mu$ and $e / h$ under different driving velocities.

between the $\gamma$ and shape of nanoparticles are known, as opposed to artificially setting them, then we can easily obtain the $e / h$ value and predict the movement pattern of nanoparticle additives by comparing the $e / h$ value with $\mu$.

Concerning the boundary and mixed lubrication systems, the lubricating fluids typically do not work well and are even squeezed out from the friction and wear regions $[14,31]$. Therefore, in our study, the presence of nanoparticle additives and their movement pattern play a key role in the removal of asperities and wear of contact surfaces. 
Figure 10, S2, and S3 (ESM) display the snapshots of worn surfaces under different tribology conditions. Under the effect of a nanoparticle shape, as reflected by the axial ratio, Fig. 10 shows that after the sliding movement of ellipsoidal nanoparticles, a regular groove appeared on the upper and lower surfaces, and a large number of $\mathrm{Cu}$ atoms were plowed from their original positions to form ridges along the two sides of the groove. Furthermore, a relatively high pileup was observed in front of the nanoparticles. A similar phenomenon was observed in the nanoscratching process [25]. Additionally, the instantaneous configurations (Fig. 4) also show that some internal defects, including dislocations and stacking faults, were nucleated in the nanoparticle contact area and propagated along $\pm 45^{\circ}$ of the sliding directions in a very limited region. This is because the applied load of $80 \mathrm{nN}$ was not sufficiently high to induce propagation of a large number of dislocations in the contact area. Hence, the elastic recovery with the removal of the load provided energy to decrease the plastic area and/ or facilitate the transformation of defects from the internal region to the surface after the nanoparticle left the area. However, for the rolling process, the formed groove, ridges, and atomic pileup were nonregular, and the worn surface atoms were distributed disorderly in the friction and wear region, especially for the nanoparticle (with higher axial ratio) closer to a sphere. Similar results are also observed in the cases with the effects of normal load and driving velocity of the upper slab, as shown in Figs. S2 and S3 (ESM). These results indicate that, as lubricant
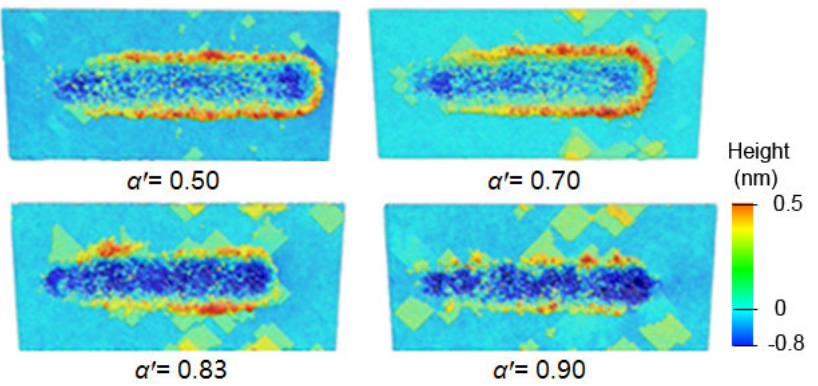

Fig. 10 Snapshots of worn surfaces with variation in axial ratio in $X O Z$ plane. The color changes from sky blue to red indicate the increasing height of atoms above the initial surface, whereas dark blue denotes a deeper scar under the initial surface. additives, sharper nanoparticles easily embed or indent into the contact surfaces and then slide. This is especially true under higher pressure and lower driving velocity, which helps reduce the surface asperities. However, it causes more severe surface damage due to the plowing action. Conversely, the nanoparticles with a shape closer to that of a sphere tend to roll between the contact surfaces, especially under lower pressure and higher driving velocity. This in turn can separate both the contact surfaces and reduce friction and wear. It is important to note that the rolling of relatively sharp nanoparticles can induce more internal defects on contact materials when the cusp comes in contact with solid surfaces under high pressure (Figs. 4 and 6). Furthermore, Fang et al. [23] reported that in a three-body abrasion, the maximum depth of the defects and scratching grooves increased with increase in the axial ratio of the ellipsoidal particle.

\section{Conclusions}

To predict and reveal the friction and wear behaviors of ellipsoidal nanoparticle additive confined between solid surfaces, a theoretical model was developed and a NEMD simulation was performed. Based on the criterion of movement pattern of particles and $e / h$ relationship for spherical nanoparticles sandwiched between solid surfaces, we developed a new $e / h$ expression for hard ellipsoidal nanoparticles. The $e / h$ is related to $\gamma, \theta, \alpha \theta$, and $\beta$ in parametric expression, which in turn are related to the normal load, material properties, and shape of the nanoparticle. Subsequently, the NEMD simulation indicated that the rolling movement of nanoparticles occurred as the axial ratio increased to 0.83 , the normal load decreased to $100 \mathrm{nN}$, and the driving velocity of the upper slab increased to $15 \mathrm{~m} / \mathrm{s}$ under certain conditions, and vice versa. By comparing the $e / h$ value calculated from the theoretical expression and coefficient of friction obtained from simulated results, it can be determined that the mathematical model of the movement criterion is in good agreement with the simulations. This in turn helps predict the movement pattern of ellipsoidal nanoparticles. 
Finally, the analysis of worn surfaces showed that the sliding movement of ellipsoidal nanoparticles causes a regular groove, ridges, and relatively high pileup in front of the nanoparticle. This resulted in severe surface damage. However, the rolling nanoparticles can separate the contact surfaces and reduce friction and wear. This study provides insights into the nanoscale lubrication and tribology behavior of nanoparticles, as lubricating additives confined between asperities.

\section{Acknowledgements}

The authors acknowledge the financial support from the National Natural Science Fundation of China (NSFC) (51905433), the National Key R\&D Program of China (No. 2018YFB0703800), and the Fundamental Research Funds for the Central Universities (No. 3102019TS0405).

Electronic Supplementary Material: Supplementary material is available in the online version of this article at https://doi.org/10.1007/s40544-020-0402-2.

Open Access This article is licensed under a Creative Commons Attribution 4.0 International License, which permits use, sharing, adaptation, distribution and reproduction in any medium or format, as long as you give appropriate credit to the original author(s) and the source, provide a link to the Creative Commons licence, and indicate if changes were made.

The images or other third party material in this article are included in the article's Creative Commons licence, unless indicated otherwise in a credit line to the material. If material is not included in the article's Creative Commons licence and your intended use is not permitted by statutory regulation or exceeds the permitted use, you will need to obtain permission directly from the copyright holder.

To view a copy of this licence, visit http://creativecommons.org/licenses/by/4.0/.

\section{References}

[1] Seymour B T, Wright R A E, Parrott A C, Gao H Y,
Martini A, Qu J, Dai S, Zhao B. Poly(alkyl methacrylate) brush-grafted silica nanoparticles as oil lubricant additives: Effects of alkyl pendant groups on oil dispersibility, stability, and lubrication property. ACS Appl Mater Interfaces 9(29): 25038-25048 (2017)

[2] Zhang X, Shen H M, Liu J, Deng S S, Li X Y, Cai Z B, Zhu M H. An efficient numerical model for predicting the torsional fretting wear considering real rough surface. Wear 344-345: 32-45 (2015)

[3] Tang Z L, Li S H. A review of recent developments of friction modifiers for liquid lubricants (2007-present). Curr Opin Solid State Mater Sci 18(3): 119-139 (2014)

[4] Spikes H. Friction modifier additives. Tribol Lett 60(1): 5 (2015)

[5] Dai W, Kheireddin B, Gao H, Liang H. Roles of nanoparticles in oil lubrication. Tribol Int 102: 88-98 (2016)

[6] Padgurskas J, Rukuiza R, Prosyčevas I, Kreivaitis R. Tribological properties of lubricant additives of $\mathrm{Fe}, \mathrm{Cu}$ and Co nanoparticles. Tribol Int 60: 224-232 (2013)

[7] Qiu S Q, Zhou Z R, Dong J X, Chen G X. Preparation of $\mathrm{Ni}$ nanoparticles and evaluation of their tribological performance as potential additives in oils. $J$ Tribol 123(3): 441-443 (2001)

[8] Yu H L, Xu Y, Shi P J, Xu B S, Wang X L, Liu Q. Tribological properties and lubricating mechanisms of $\mathrm{Cu}$ nanoparticles in lubricant. Trans Nonferr Met Soc China 18(3): 636-641 (2008)

[9] Zhao J H, Yang G B, Zhang C L, Zhang Y J, Zhang S M, Zhang P Y. Synthesis of water-soluble $\mathrm{Cu}$ nanoparticles and evaluation of their tribological properties and thermal conductivity as a water-based additive. Friction 7(3): 246-259 (2019)

[10] Battez A H, González R, Viesca J L, Fernández J E, Fernández J M D, Machado A, Chou R, Riba J. CuO, $\mathrm{ZrO}_{2}$ and $\mathrm{ZnO}$ nanoparticles as antiwear additive in oil lubricants. Wear 265(3-4): 422-428 (2008)

[11] Cortes V, Sanchez K, Gonzalez R, Alcoutlabi M, Ortega $\mathrm{J}$ A. The performance of $\mathrm{SiO}_{2}$ and $\mathrm{TiO}_{2}$ nanoparticles as lubricant additives in sunflower oil. Lubricants 8(1): 10 (2020)

[12] Jiao D, Zheng S H, Wang Y Z, Guan R F, Cao B Q. The tribology properties of alumina/silica composite nanoparticles as lubricant additives. Appl Surf Sci 257(13): 5720-5725 (2011)

[13] Wu Y Y, Tsui W C, Liu T C. Experimental analysis of tribological properties of lubricating oils with 
nanoparticle additives. Wear 262(7-8): 819-825 (2007)

[14] Ewen J P, Gattinoni C, Thakkar F M, Morgan N, Spikes $\mathrm{H}$ A, Dini D. Nonequilibrium molecular dynamics investigation of the reduction in friction and wear by carbon nanoparticles between iron surfaces. Tribol Lett 63(3): 38 (2016)

[15] Golchin A, Wikner A, Emami N. An investigation into tribological behaviour of multi-walled carbon nanotube/ graphene oxide reinforced UHMWPE in water lubricated contacts. Tribol Int 95: 156-161 (2016)

[16] Gupta B K, Bhushan B. Fullerence particles as an additive to liquid lubricants and greases for low friction and wear. Lubr Eng 50(7): 524-528 (1994)

[17] Ginzburg B M, Kireenko O F, Shepelevskii A A, Shibaev L A, Tochilnikov D G, Leksovskii A M. Thermal and tribological properties of fullerene-containing composite systems. Part 2. Formation of tribo-polymer films during boundary sliding friction in the presence of fullerene $\mathrm{C}_{60}$. J Macromol Sci, Part B 44(1): 93-115 (2005)

[18] Xu T, Zhao J Z, Xu K. The ball-bearing effect of diamond nanoparticles as an oil additive. J Phys D: Appl Phys 29(11): 2932-2937 (1996)

[19] Liang Q, Tsui O K C, Xu Y B, Li H N, Xiao X D. Effect of $\mathrm{C}_{60}$ molecular rotation on nanotribology. Phys Rev Lett 90(14): 146102 (2003)

[20] Coffey T, Krim J. $\mathrm{C}_{60}$ molecular bearings and the phenomenon of nanomapping. Phys Rev Lett 96(18): 186104 (2006)

[21] Lee K, Hwang Y, Cheong S, Choi Y, Kwon L, Lee J, Kim S H. Understanding the role of nanoparticles in nano-oil lubrication. Tribol Lett 35(2): 127-131 (2009)

[22] Shi J Q, Fang L, Sun K. Friction and wear reduction via tuning nanoparticle shape under low humidity conditions: A nonequilibrium molecular dynamics simulation. Comput Mater Sci 154: 499-507 (2018)

[23] Fang L, Sun K, Shi J Q, Zhu X Z, Zhang Y N, Chen J, Sun J P, Han J. Movement patterns of ellipsoidal particles with different axial ratios in three-body abrasion of monocrystalline copper: A large scale molecular dynamics study. RSC Adv 7(43): 26790-26800 (2017)

[24] Zhang X, Wang Z J, Shen H M, Wang Q J. An efficient model for the frictional contact between two multiferroic bodies. Int J Solids Struct 130-131: 133-152 (2018)

[25] Shi J Q, Chen J, Fang L, Sun K, Sun J P, Han J. Atomistic scale nanoscratching behavior of monocrystalline $\mathrm{Cu}$ influenced by water film in CMP process. Appl Surf Sci 435: 983-992 (2018)
[26] Shi J Q, Fang L, Sun K, Peng W X, Ghen J, Zhang M. Surface removal of a copper thin film in an ultrathin water environment by a molecular dynamics study. Friction 8(2): 323-334 (2020)

[27] Sun J P, Fang L, Han J, Han Y, Chen H W, Sun K. Abrasive wear of nanoscale single crystal silicon. Wear 307(1-2): 119-126 (2013)

[28] Fang L, Kong X L, Su J Y, Zhou Q D. Movement patterns of abrasive particles in three-body abrasion. Wear 162-164: 782-789 (1993)

[29] Shi J Q, Wei X Q, Chen J, Sun K, Fang L. Influence of abrasive shape on the abrasion and phase transformation of monocrystalline silicon. Crystals 8(1): 32 (2018)

[30] Plimpton S. Fast parallel algorithms for short-range molecular dynamics. J Comput Phys 117(1): 1-19 (1995)

[31] Persson B, Mugele F. Squeeze-out and wear: Fundamental principles and applications. J Phys: Condens Matter 16(10): R295-R355 (2004)

[32] Ewen J P, Gattinoni C, Morgan N, Spikes H A, Dini D. Nonequilibrium molecular dynamics simulations of organic friction modifiers adsorbed on iron oxide surfaces. Langmuir 32(18): 4450-4463 (2016)

[33] Mishin Y, Mehl M J, Papaconstantopoulos D A, Voter A F, Kress J D. Structural stability and lattice defects in copper: $A b$ initio, tight-binding, and embedded-atom calculations. Phys Rev B 63(22): 224106 (2001)

[34] Morse P M. Diatomic molecules according to the wave mechanics. II. Vibrational levels. Phys Rev 34(1): 57-64 (1929)

[35] Shi J Q, Chen J, Sun K, Sun J P, Han J, Fang L. Water film facilitating plastic deformation of $\mathrm{Cu}$ thin film under different nanoindentation modes: A molecular dynamics study. Mater Chem Phys 198: 177-185 (2017)

[36] Shi J Q, Zhang Y N, Sun K, Fang L. Effect of water film on the plastic deformation of monocrystalline copper. RSC Adv 6(99): 96824-96831 (2016)

[37] Ren J Q, Zhao J S, Dong Z G, Liu P K. Molecular dynamics study on the mechanism of AFM-based nanoscratching process with water-layer lubrication. Appl Surf Sci 346: 84-98 (2015)

[38] Boda D, Henderson D. The effects of deviations from Lorentz-Berthelot rules on the properties of a simple mixture. Mol Phys 106(20): 2367-2370 (2008)

[39] Al-Matar A K, Rockstraw D A. A generating equation for mixing rules and two new mixing rules for interatomic potential energy parameters. J Comput Chem 25(5): 660-668 (2004) 


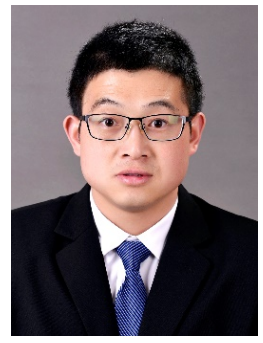

Junqin SHI. He received his M.S. degree in physics in 2015 from China University of Petroleum (East China). After then, he received his Ph.D. degree in materials science and engineering in 2018 at Xi'an Jiaotong University. His current position is an associate professor in the Center of

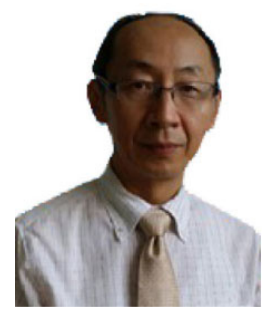

Liang FANG. He received his M.S. and Ph.D. degrees in materials science and engineering from Xi'an Jiaotong University and Harbin Institute of Technology, China, in 1984 and 1991, respectively. His current position is a professor in
Advanced Lubrication and Seal Materials, State Key Laboratory of Solidification Processing at Northwestern Polytechnical University, China. His research interests include plastic deformation, friction and wear behavior of crystal materials, lubrication at extreme conditions, and the computational materials science.

the State Key Laboratory for Mechanical Behavior of Materials at Xi'an Jiaotong University and the dean of School of Mechanical and Electrical Engineering in Xiamen University Tan Kah Kee College. His research areas cover the tribology and processing of metallic materials. 